\title{
Graphical Analysis of Interactions between Oxidation-Reduction Sites in Two Site Oxidation-Reduction Proteins ${ }^{1}$
}

\author{
Michael E. O'Donnell ${ }^{2}$ and Charles H. Williams, JR. \\ Veterans Administration Medical Center and Department of Biological Chemistry, \\ The University of Michigan, Ann Arbor, Michigan 48105
}

Received June 22, 1983

\begin{abstract}
Many enzymes that catalyze electron-transfer reaction contain multiple oxidation-reduction centers (sites). The oxidation-reduction potential of one site as well as the kinetics of electron transfer through this site may be altered by the state of reduction of a neighboring site. Oxidationreduction site interactions may be mechanistically important and quantitation of site interactions would aid the interpretation of thermodynamic data and possibly kinetic data. A graphical means to detect and quantitate interactions between oxidation-reduction sites from oxidation-reduction equilibrium data (type $A+B \frown C+D$ ) is described and has its roots in the Scatchard analysis of ligand binding equilibria (type $\mathrm{A}+\mathrm{B} \rightarrow \mathrm{C}$ ). Oxidation-reduction sites often have distinct physical properties allowing the titration behavior of specific sites to be monitored. Equilibrium measurements on specific sites of a two site protein allow a further analysis of the data which can be combined with the oxidation-reduction Scatchard analysis to solve for all four specific site equilibrium constants. Ligand binding systems can usually measure only total site binding and simplifying assumptions of identical sites or noninteracting sites are required to solve for the site specific equilibrium constants. Thus, specific site equilibrium measurements offer a distinct advantage over total site measurements. The principles of the method are illustrated by applying the graphical analysis to the two site protein, thioredoxin reductase, which contains an oxidation-reduction active site disulfide in addition to FAD. The specific site oxidation-reduction midpoint potentials $\left(E_{\mathrm{m}}\right)$ of the FAD and disulfide couples of thioredoxin reductase at $\mathrm{pH} 6.0$, $12^{\circ} \mathrm{C}$, were found to be FAD/FADH ${ }_{2}$-enzyme-(S $)_{2}=-0.183 \mathrm{~V}$, FAD/FADH ${ }_{2}$-enzyme-(SH $)_{2}$ $=-0.199 \mathrm{~V},(\mathrm{FAD})$-enzyme-(S) $)_{2} /(\mathrm{SH})_{2}=-0.202 \mathrm{~V}$, and $\left(\mathrm{FADH}_{2}\right)$-enzyme- $(\mathrm{S})_{2} /(\mathrm{SH})_{2}=-0.218$ $\mathrm{V}$. Hence, at $\mathrm{pH} 6.0$, the FAD and disulfide sites of thioredoxin reductase have $E_{\mathrm{m}}$ values that differ by approximately $0.019 \mathrm{~V}$ and have a negative interaction of about $0.016 \mathrm{~V}$.

KEY WORDS: oxidation/reduction; Scatchard analysis; enzymes; flavoprotein; physical techniques; redox potentials, thioredoxin reductase.
\end{abstract}

The biochemical literature cites many examples of allosteric and coopcrative interactions among ligand binding sites in multiple site ligand binding proteins $(1,2)$. Many enzymes which catalyze electron transfer processes contain multiple oxidation-reduction sites. The field of biological oxidations has not, in general, investigated interactions

\footnotetext{
'This paper was taken from a thesis submitted by M. E. O'Donnell to the Graduate School of the University of Michigan in partial fulfillment of the requirements for the Ph.D. degree.

${ }^{2}$ Present address: Department of Biochemistry, Stanford University School of Medicine, Stanford, California 94305.
}

among oxidation-reduction sites. Quantitation of site interactions would aid the interpretation of thermodynamic data. Further, interactions among oxidation-reduction sites may be reflected in kinetic data since the rate of electron transfer to one site may be affected by the oxidation-reduction state of a neighboring site.

Interactions among oxidation-reduction sites have been investigated in cytochrome oxidase (3-8) and in other proteins $(9,10)$. Two methods are used in these studies to analyze the interactions between sites. One method uses a plot of a signal representing the frac- 
tional reduction of one oxidation-reduction site versus a signal that represents the fractional reduction of another site or all the sites combined. A model that assigns oxidationreduction potential differences between the sites is fit to the data points. The data for this plot are usually obtained from titrations using a titrant having a low oxidation-reduction potential relative to the protein couples (i.e., sodium dithionite) since this analysis does not require knowing the equilibrium concentrations of oxidized and reduced titrant. Hence, the oxidation-reduction midpoint potentials $\left(E_{\mathrm{m}}\right)$ for the protein couples are not usually obtained. A further disadvantage of this method is the inability to detect interactions among initially identical sites (4). Another method to analyze interactions between oxidation-reduction sites is the Nernst analysis. Equilibrium concentrations of oxidized and reduced species of the protein as well as the titrant are required in the Nernst analysis and $E_{\mathrm{m}}$ values for the protein couples are obtained. However, in the Nernst analysis, equilibrium data at very high and very low levels of reduction are required to quantitate interactions between sites and the difference in $E_{\mathrm{m}}$ between sites (site heterogeneity). Accurate equilibrium data at high and low levels of reduction are usually difficult to collect.

The Nernst plot of oxidation-reduction equilibria is analogous to the Hill plot of ligand binding. In a detailed comparison of the Hill and Scatchard plots, the Scatchard plot has been shown to be more useful than the Hill plot for quantitative analysis of nonidentity between the sites and negative interactions between the sites (11). Thus, the validity of a graphical method to analyze oxidation-reduction equilibria that is analogous to the Scatchard analysis of ligand binding is examined.

In ligand binding systems, the binding of ligand to specific sites is not generally measurable. Thus, analysis of even the simplest case, a two site protein, requires assumptions of no interactions between sites or of identical sites to solve for the site specific equilibrium constants. Oxidation-reduction centers often have characteristic physical properties which permit measurements of the equilibrium concentrations of oxidized and reduced species of specific sites. Specific site equilibrium data yields further information on site heterogeneity and site interactions and the site specific equilibrium constants of a two site system can be solved. The principles of the method are illustrated by considering a two-site system in detail and applying the analysis to thioredoxin reductase, an enzyme that contains two oxidation-reduction sites.

\section{MATERIALS AND METHODS}

Thioredoxin reductase was purified from E. coli by a modification of the procedure of Pigiet and Conley (12) except $1.0 \mathrm{M} \mathrm{NaCl}$ was used in place of NADPH to elute the enzyme from the $2^{\prime}, 5^{\prime}$-ADP affinity column since the aerobic turnover of NADPH leads to peroxide mediated enzyme modification. ${ }^{3} \mathrm{NAD}^{+}$and NADH were purchased from Sigma. Anaerobic spectral titrations of thioredoxin reductase with NADH were performed using approximately $17 \mu \mathrm{M}$ enzyme in $0.1 \mathrm{M}$ $\mathrm{NaH}_{2} \mathrm{PO}_{4}-\mathrm{K}_{2} \mathrm{HPO}_{4}-0.3 \mathrm{mM}$ EDTA, pH 6.0, at $12^{\circ} \mathrm{C}$. The anaerobic methods were described elsewhere (13). Absorbance measurements were performed using a Cary $118 \mathrm{C}$ spectrophotometer. Measurements of $\mathrm{pH}$ were performed using a Beckman Model $3500 \mathrm{pH}$ meter and Thomas 4094-L15 combination electrode. The equilibrium concentrations of enzyme FAD and enzyme $\mathrm{FADH}_{2}$ were calculated from the absorbance at $456 \mathrm{~nm}$ (13). The equilibrium concentration of NADH was obtained from an isosbestic wavelength in the reduction of thioredoxin reductase and the concentrations of $\mathrm{NAD}^{+}$and oxidized and reduced disulfide of thioredoxin reductase were obtained by difference (13). Corrections to the data for FAD semiquinone were described elsewhere (13). The criteria of negligible binding between oxidation-reduction species of

\footnotetext{
${ }^{3}$ O'Donnell, M. E., and Williams, C. H., Jr., unpublished data.
} 
titrant and enzyme and the condition of equilibrium among oxidized and reduced protein and titrant species were fulfilled as described elsewhere (13). The value of $E_{\mathrm{m}}$ for the $\mathrm{NAD}^{+} /$ $\mathrm{NADH}$ couple was taken to be $-0.315 \mathrm{~V}$ at $\mathrm{pH} 7.0$ and $25^{\circ} \mathrm{C}$ as given by Clark (14). Applying the temperature correction factor of $0.0013 \mathrm{~V} /{ }^{\circ} \mathrm{C}$ (14), and slope, $\Delta E_{\mathrm{m}} / \Delta \mathrm{pH}$, of $0.0283 \mathrm{~V} / \mathrm{pH}$ unit at $12^{\circ} \mathrm{C}(14)$, the $E_{\mathrm{m}}$ value for the $\mathrm{NAD}^{+} / \mathrm{NADH}$ couple is $-0.270 \mathrm{~V}$ at pH 6.0 and $12^{\circ} \mathrm{C}$.

The experimental data were fitted to equations using a computer program (15) adapted by the Statistics Research Laboratory, The University of Michigan.

\section{RESULTS}

\section{Analogy of Oxidation-Reduction Equilibria to Ligand Binding Equilibria}

Ligand binding. The simple binding of one ligand molecule to a protein is described by

$$
P+L \stackrel{K_{a}}{\leftarrow} P-L
$$

where $P$ is protein, $L$ is unbound ligand, $P$ $L$ is protein-ligand complex, and $K_{a}$ is the equilibrium constant for complex formation. The number of moles of ligand bound per mole of protein, $\bar{v}$, is related to Eq. [1] by

$$
\bar{v}=\frac{P-L}{P+P-L}=\frac{K_{a}[L]}{1+K_{a}[L]} .
$$

Several linear transformations of Eq. [2] have been derived which allow the graphical evaluation of $K_{a}$. The Scatchard transformation of Eq. [2] has received widespread use in the analysis of ligand binding equilibrium data and is given by (16)

$$
\bar{v} /[L]=-\bar{v} \mathbf{K}_{a}+\mathbf{K}_{a} .
$$

Oxidation-reduction. A simple biological oxidation-reduction reaction is described by

$$
P_{\text {ox }}+N_{\mathrm{r}} \stackrel{K_{\text {eq }}}{\sim} P_{\mathrm{r}}+N_{\mathrm{ox}} \text {, }
$$

where $P_{\mathrm{ox}}$ and $P_{\mathrm{r}}$ are oxidized and reduced species of protein, respectively, $N_{\mathrm{ox}}$ and $N_{\mathrm{r}}$ are oxidized and reduced forms of titrant, re- spectively, and $K_{\text {eq }}$ is the equilibrium constant for the reaction. The fractional level of reduction (i.e., the number of moles of reduced sites per mole of protein), $\bar{v}$, is related to Eq. [4] by

$$
\bar{v}=\frac{P_{\mathrm{r}}}{P_{\mathrm{ox}}+P_{\mathrm{r}}}=\frac{K_{\mathrm{eq}}\left[N_{\mathrm{r}}\right] /\left[N_{\mathrm{ox}}\right]}{1+K_{\mathrm{eq}}\left[N_{\mathrm{r}}\right] /\left[N_{\mathrm{ox}}\right]} .
$$

Equation 5 is analogous to Eq. [2], i.e., substitution of $\left[N_{\mathrm{r}}\right] /\left[N_{\mathrm{ox}}\right]$ for $[L]$. The analogy is intuitively reasonable. The concentration of free ligand is a driving force in the complexation equilibrium of Eq. [1]. Similarly, the ratio of the concentration of reduced titrant to oxidized titrant is the driving force in the reduction of protein in the equilibrium of Eq. [4]. It is important to note that oxidationreduction potentials measured with an electrode can be converted into Eq. [5] through the Nernst equation.

It is apparent from the analogy between Eqs. [2] and [5] that the theoretical framework for analyzing ligand binding equilibria can be applied to the analysis of oxidation-reduction equilibria, or, for that matter, any equilibrium of the type $\mathrm{A}+\mathrm{B} \hookrightarrow \mathrm{C}+\mathrm{D}$. The transformation of Eq. [5] into the analogous Scatchard equation of ligand binding is shown below and will be refered to as the Redox Scatchard equation:

$$
\bar{v}\left[N_{\mathrm{ox}}\right] /\left[N_{\mathrm{r}}\right]=-K_{\mathrm{eq}} \bar{v}+K_{\mathrm{eq}} .
$$

\section{Analysis of Interactions between the Oxidation-Reduction Sites of a Two Site Protein}

The titration behavior of a protein containing two oxidation-reduction sites can be described by the linked equilibria shown in Fig. 1. The average number of reduced sites per protein molecule, $\bar{v}$, is given by

$$
\bar{v}=\frac{\left[{ }_{\mathrm{ox}} P_{\mathrm{red}}\right]+\left[{ }_{\mathrm{red}} P_{\mathrm{ox}}\right]+2\left[{ }_{\mathrm{red}} P_{\mathrm{red}}\right]}{\left.\left.\left[{ }_{\mathrm{ox}} P_{\mathrm{ox}}\right]+\left[{ }_{\mathrm{ox}} P_{\mathrm{red}}\right]+{ }_{\mathrm{red}} P_{\mathrm{ox}}\right]+{ }_{\mathrm{red}} P_{\mathrm{red}}\right]} .
$$

Substitution of the site specific equilibrium constants and titrant species for the protein 


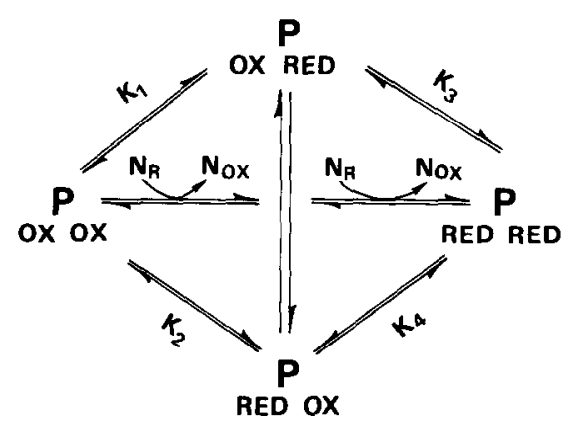

FIG. 1. Equilibria between the four oxidation-reduction states of a two-site protein and oxidized and reduced forms of titrant. $P$, protein; $N$, titrant; and ox and red, oxidized or reduced state of the protein sites and titrant respectively.

species yields

$$
\begin{aligned}
& K_{1}\left[N_{\mathrm{r}}\right] /\left[N_{\mathrm{ox}}\right] \\
& \bar{v}=\frac{+K_{2}\left[N_{\mathrm{r}}\right] /\left[N_{\mathrm{ox}}\right]+}{2 K_{1} K_{3}\left(\left[N_{\mathrm{r}}\right] /\left[N_{\mathrm{ox}}\right]\right)^{2}} \\
& 1+K_{1}\left[N_{\mathrm{r}}\right] /\left[N_{\mathrm{ox}}\right]+K_{2}\left[N_{\mathrm{r}}\right] /\left[N_{\mathrm{ox}}\right] \\
&+K_{1} K_{3}\left(\left[N_{\mathrm{r}}\right] /\left[N_{\mathrm{ox}}\right]\right)^{2}
\end{aligned}
$$

Equation [9] is derived from Eq. [8] by a mathematical transformation known as separation into partial fractions $(17,18)$ :

$$
\begin{aligned}
\bar{v}=\frac{K_{\alpha}\left[N_{\mathrm{r}}\right] /\left[N_{\mathrm{ox}}\right]}{1+K_{\alpha}\left[N_{\mathrm{r}}\right] /\left[N_{\mathrm{ox}}\right]} & \\
& +\frac{K_{\beta}\left[N_{\mathrm{r}}\right] /\left[N_{\mathrm{ox}}\right]}{1+K_{\beta}\left[N_{\mathrm{r}}\right] /\left[N_{\mathrm{ox}}\right]} .
\end{aligned}
$$

Equation [9] is analogous to transformation of equations for the Scatchard analysis of multiple ligand binding sites (17). The phenomenological constants of Eq. [9], $K_{\alpha}$ and $K_{\beta}$, are expressed in terms of the site specific equilibrium constants by

$$
\begin{aligned}
K_{\alpha}= & 1 / 2\left(K_{1}+K_{2}\right) \\
& +1 / 2\left[\left(K_{1}+K_{2}\right)^{2}-4 K_{1} K_{3}\right]^{1 / 2} \\
K_{\beta}= & 1 / 2\left(K_{1}+K_{2}\right) \\
& -1 / 2\left[\left(K_{1}+K_{2}\right)^{2}-4 K_{1} K_{3}\right]^{1 / 2}
\end{aligned}
$$

It is of extreme importance to note that the phenomenological constants, $K_{\alpha}$ and $K_{p}$, do not generally correspond to site specific equilibrium constants but are functions of the site specific equilibrium constants. This is a source of much confusion in published interpretations of multiple ligand binding equilibria.

Transformation of Eq. [9] into the Redox Scatchard equation does not yield a linear relationship. This is analogous to the transformation of equations for multiple ligand binding sites into the Scatchard plot function $(19,20)$. Thus, the data are plotted according to the Redox Scatchard plot function:

$$
\bar{v} \frac{\left[N_{\mathrm{ox}}\right]}{\left[N_{\mathrm{r}}\right]}=f(\bar{v}) .
$$

The graphical patterns of the Redox Scatchard plot for a two-site protein are shown in Fig. 2 for the cases of identical, nonidentical, negative interacting, or noninteracting sites. The limiting slopes and intercepts for the Redox Scatchard plot of Fig. 2 have been derived in terms of the phenomenological constants and are summarized in Table 1.

The first step toward solving the site specific equilibrium constants of Fig. 1 is to obtain the values of the phenomenological constants from the Redox Scatchard plot (Fig. 2). In the case of identical/noninteracting sites the Redox Scatchard plot is linear (Fig. 2A) and $K_{\alpha}$ and $K_{\beta}$ are obtained from the slope. For nonidentical and/or interacting sites, the Redox Scatchard plot is curved (Fig. 2B). The limiting slopes and intercepts of a curved Redox Scatchard plot can be expressed in terms of the phenomenological constants (Table 1). Thus $K_{\alpha}$ and $K_{\beta}$ can be obtained from the slopes and intercepts of a curved Redox Scatchard plot. A computer program which fits values for the phenomenological constants to ligand binding equilibrium data has been developed and is available (15). The computer analysis can be directly applied to oxidation-reduction equilibrium data by substituting $\left[N_{\mathrm{ox}}\right] /\left[N_{\mathrm{r}}\right]$ for $[L]$. A computer-assisted fit of the equilibrium data avoids the subjective bias often associated with hand-drawn slopes and intercepts and thus is an especially valuable method to obtain the values of the phenomenological constants from a curved Redox Scatchard plot.

Thrce site spccific equilibrium constants are 


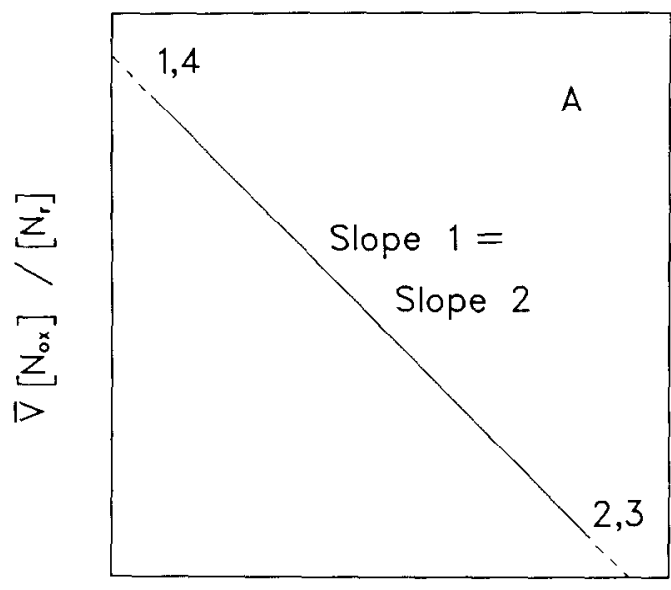

$\bar{V}$

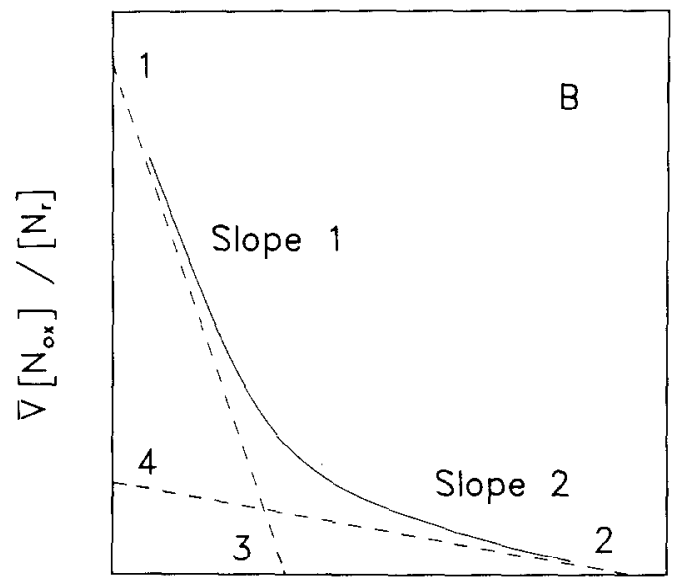

$\bar{\nabla}$

FIG. 2. Redox Scatchard plots for a two-site protein having identical or nonidentical $E_{\mathrm{m}}$ values and either no interaction between the sites or a negative interaction. The slopes and intercepts are indicated on the plots. (A) Case of noninteracting/identical sites. (B) Cases of negative interacting/identical, noninteracting/ nonidentical sites, and negative interacting/nonidentical sites.

required to fully describe the linked equilibrium of the two site protein shown in Fig. 1 (i.e., the remaining equilibrium constant is defined by the relationship $K_{1} K_{3}=K_{2} K_{4}$ ). The two phenomenological constants obtained from the Redox Scatchard analysis are each functions of three site specific equilibrium constants. Thus, a third relationship between the site specific equilibrium constants is required to solve for the three site specific constants. In ligand binding systems, only total site binding can be measured. Thus, for curved Scatchard plots, simplifying assumptions must be made to obtain the site specific equilibrium constants from the two expressions for $K_{\alpha}$ and $K_{\beta}$. For example, the assumption of identical sites $\left(K_{1}=K_{2}, K_{3}=K_{4}\right)$ simplifies the expressions for $K_{\alpha}$ and $K_{\beta}$ into functions of only two site specific equilibrium constants. The two site specific constants are obtained from simultaneous solution of the simplified expressions for $K_{\alpha}$ and $K_{\beta}$. Likewise, the assumption of noninteracting sites $\left(K_{1}=K_{4}, K_{2}=K_{3}\right)$ simplifies the expression for $K_{\alpha}$ and $K_{\beta}$ into functions of two site specific constants from which the values for the two site specific equilibrium constants can be solved.
Oxidation-reduction sites are often small organic molecules or transition metal atoms which have distinct physical properties. Physical properties characteristic to specific sites can be used to monitor the fractional level of reduction of individual sites during a titration. A third relationship among the site specific equilibrium constants is obtained from site specific data allowing all four site specific equilibrium constants of Fig. 1 to be solved without the need for assumptions.

The expressions for the fractional reduction of the individual sites of the two site protein in Fig. 1 are given by

$$
\begin{aligned}
& \bar{v}_{1}=\frac{\left[{ }_{\mathrm{ox}} P_{\mathrm{red}}\right]+\left[{ }_{\mathrm{red}} P_{\mathrm{red}}\right]}{\left.\left.\left[{ }_{\mathrm{ox}} P_{\mathrm{ox}}\right]+\left[{ }_{\mathrm{ox}} P_{\mathrm{red}}\right]+{ }_{\mathrm{red} d} P_{\mathrm{ox}}\right]+{ }_{\mathrm{red}} P_{\mathrm{red}}\right]} \\
& \bar{v}_{2}=\frac{\left[{ }_{\mathrm{red}} P_{\mathrm{ox}}\right]+\left[{ }_{\mathrm{red}} P_{\mathrm{red}}\right]}{\left.\left[{ }_{\mathrm{ox}} P_{\mathrm{ox}}\right]+\left[{ }_{\mathrm{ox}} P_{\mathrm{red}}\right]+{ }_{\mathrm{red}} P_{\mathrm{ox}}\right]+\left[{ }_{\mathrm{red}} P_{\mathrm{red}}\right]} .
\end{aligned}
$$

Dividing $\bar{v}_{1}$ by $\bar{v}_{2}$ :

$$
\frac{\bar{v}_{1}}{\bar{v}_{2}}=\frac{\left[\mathrm{ox} P_{\text {red }}\right]+\left[{ }_{\text {red }} P_{\text {red }}\right]}{\left[\text { red } P_{\mathrm{ox}}\right]+\left[{ }_{\text {red }} P_{\text {red }}\right]}
$$




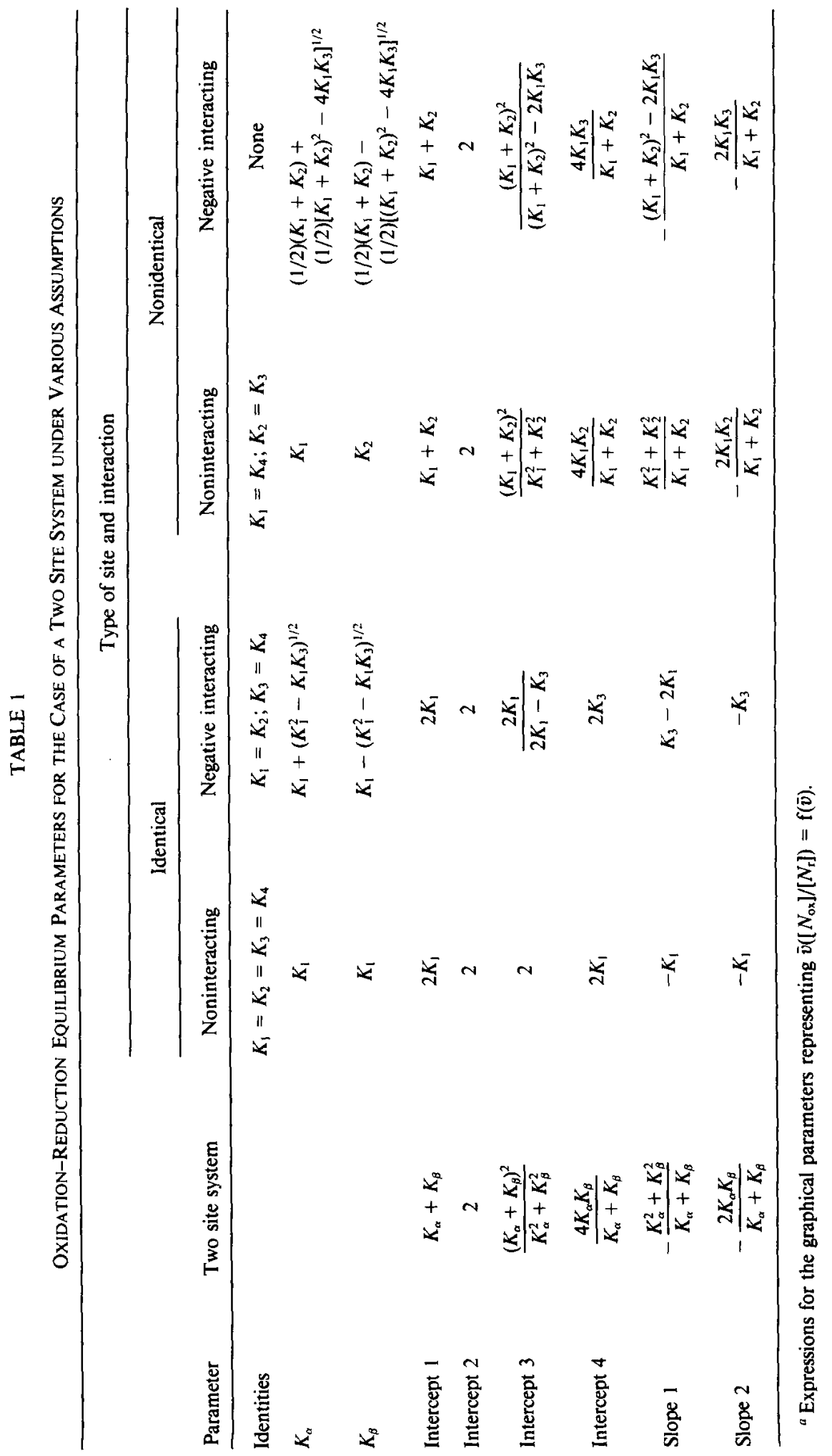


Substitution of the site specific equilibrium constants and titrant species for the protein species and rearrangement of the resulting equation yields

$$
\frac{\left(\bar{v}_{1}-\bar{v}_{2}\right)\left[N_{\mathrm{r}}\right]}{\bar{v}_{2}\left[N_{\mathrm{ox}}\right]}=-\frac{\bar{v}_{1} \mathrm{~K}_{2}}{\bar{v}_{2} \mathrm{~K}_{1} \mathrm{~K}_{3}}+\frac{1}{K_{3}} .
$$

Equation [14] is a linear relationship and can be plotted as shown in Fig. 3. The value of $K_{3}$ is the reciprocal of the intercept on the ordinate. The value of $K_{3}$ together with the values of $K_{\alpha}$ and $K_{\beta}$ are substituted into Eqs. $[10 \mathrm{~A}]$ and $[10 \mathrm{~B}]$ to solve for two other site specific equilibrium constants. For the linked equilibrium of Fig. 1, the identity $K_{1} K_{3}$ $=K_{2} K_{4}$, allows the remaining site specific equilibrium constant to be calculated.

The pattern of the Redox Scatchard plot for each of the four cases of a two-site system involving site heterogeneity and site interactions are analyzed below. Expressions for the slopes and intercepts in terms of phenomenological constants and site specific equilibrium constants for each of the cases are summarized in Table 1. In addition, the principles

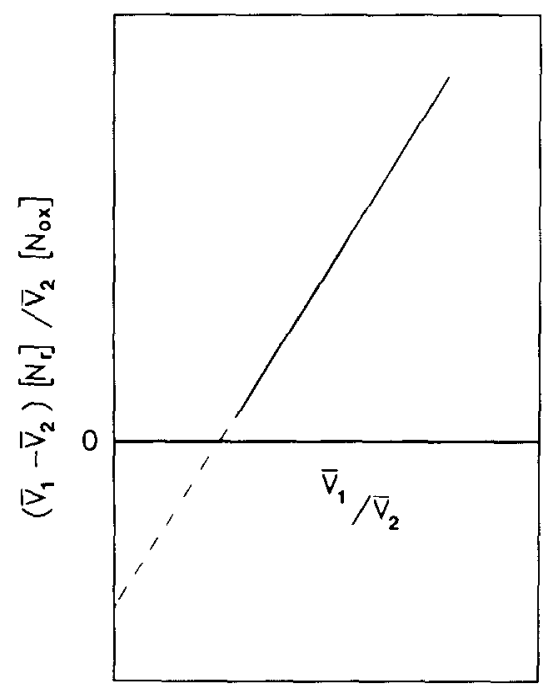

FIG. 3. Plot of site specific equilibrium data according to Eq. [14] for the case of negative interacting/nonidentical sites of a two site protein. The values of the fractional level of reduction of sites 1 and 2 are represented by $\bar{v}_{1}$ and $\bar{v}_{2}$, respectively. The oxidized and reduced form of titrant are $N_{\mathrm{ux}}$ and $N_{1}$, respectively. involved in the Redox Scatchard analysis will be illustrated by applying the method to data from the equilibrium between the $\mathrm{NAD}^{+} /$ $\mathrm{NADH}$ couple and the couples of thioredoxin reductase, a two site protein.

Noninteracting/identical sites. Here, $K_{1}$ $=K_{2}=K_{3}=K_{4}$ and the expressions for the phenomenological constants reduce to $K_{\alpha}$ $=K_{\beta}=K_{1}$. The Redox Scatchard plot for this case is shown in Fig. 2A. The plot is a straight line and is interpreted in an analogous manner to the plot for a single site with the exception that Intercept 2 (Intercept 3 ) is 2 instead of 1 and Intercept 1 (Intercept 4) is twice the value of the site specific equilibrium constant.

Noninteracting/nonidentical sites. For this case, $K_{1}=K_{4}, K_{2}=K_{3}$ and the expressions for the phenomenological constants simplify to $K_{\alpha}=K_{1}=K_{4}$; and $K_{\beta}=K_{2}=K_{3}$. The Redox Scatchard plot is a curved line which is concave upward as shown in Fig. 2B. As in the case of noninteracting/identical sites, the phenomenological constants are site specific equilibrium constants. Fxpressions for the slopes of the Redox Scatchard plot in terms of site specific equilibrium constants are functions of both $K_{1}$ and $K_{2}$ and thus the two slopes must be solved simultaneously to obtain the site specific equilibrium constants.

Extrapolation of the initial slope to the abscissa (Intercept 3) represents the maximum number of sites having the higher oxidationreduction potential, Intercept 2 gives the total number of sites, and Intercept 1 divided by Intercept 2 yields the average equilibrium constant (i.e., average value of $E_{\mathrm{m}}$ ) for reduction of the first site.

Direct equilibrium measurements of the fractional level of reduction of each site greatly simplifies the analysis of the site specific equilibrium constants. For the case of noninteracting/nonidentical sites, each site is in equilibrium with the titrant species independent of the state of the other site and the Redox Scatchard plot of the individual sites are each a straight line (i.e., Fig. 2A). The site specific equilibrium constants are obtained by analysis of the individual Redox Scatchard plots ac- 
cording to Eq. [6], the equation for a one-site protein. Thus, site specific information obviates the necessity for the curve fitting method.

Negative interacting/identical sites. For this case, $K_{1}=K_{2}, K_{3}=K_{4}$ and the phenomenological constants reduce to $K_{\alpha}=K_{1}$ $+\left(K_{1}^{2}-K_{1} K_{3}\right)^{1 / 2}$ and $K_{\beta}=K_{1}-\left(K_{1}^{2}-K_{1} K_{3}\right)^{1 / 2}$. In contrast to the two cases discussed above, the phenomenological constants are not site specific equilibrium constants. For this case, the phenomenological constants are functions of a combination of the site specific equilibrium constants and are solved simultaneously to yield the site specific equilibrium constants (i.e., $K_{\alpha}+K_{\beta}=2 K_{1}$ and $K_{\alpha} K_{\beta}=K_{1} K_{3}$ ). The Redox Scatchard plot is a curved line which is concave upward for a negative interaction (Fig. 2B).

It is important to note that the shapes of the Redox Scatchard plots for the cases of negative interacting/identical sites and for noninteracting/nonidentical sites are indistinguishable (Fig. 2B). Thus, for plots of these cases whose curves are superimposable, the phenomenological constants will be identical but the values for the site specific equilibrium constants solved from the phenomenological constants will be different since their expressions in terms of site specific equilibrium constants differ for the two cases. For the case of negative interacting/identical sites, the limiting slope at high values of fractional reduction is the site specific equilibrium constant for one site when the other site is reduced. The initial limiting slope is more complex and is a function of the site specific equilibrium constant for one site when the other site is oxidized, $K_{1}$, and the site specific equilibrium constant for one site when the other site is reduced, $K_{3}$. For an especially strong negative interaction bctwcen oxidation-reduction sites, $K_{3}$ is small with respect to $K_{1}$ and the initial limiting slope is twice the value of $-K_{1}$. Extrapolation of the initial slope to the abscissa (Intercept 3) represents the strength of the interaction between the sites. For a strongly negative interaction between the sites, $K_{3}$ is small relative to $K_{1}$ and Intercept $3=1$. If there is no interaction between the sites, $K_{1}$ $=K_{3}$ and Intercept $3=2$. Thus, for this case, the value of Intercept 3 is qualitatively similar to the value of the slope of the Nernst plot (i.e., the analog of the Hill coefficient of ligand binding). Intercept 2 gives the total number of sites and Intercept 1 divided by Intercept 2 yields the average value of $E_{\mathrm{m}}$ for reduction of the first site.

Negative interacting/nonidentical sites. In this case, there are no identities and the phenomenological constants retain the form of Eqs. [10A] and [10B]. The Redox Scatchard plot for this case is a curve which is concave upward (Fig. 2B). As in each of the simpler cases, Intercept 2 and Intercept 1 yield the total number of sites and the average $E_{\mathrm{m}}$ value for reduction of the first site. The other intercepts and the slopes do not allow qualitative interpretations since they are functions of three site specific equilibrium constants (Table 1).

In the case of negative interacting/nonidentical sites, the phenomenological constants are functions of three site specific equilibrium constants, and cannot be solved from the phenomenological constants alone. The Redox Scatchard plots of the individual sites are dissimilar curves which yield no information other than to confirm that the sites have unequal $E_{\mathrm{m}}$ values and have a negative interaction between them. Thus, individual site oxidation-reduction equilibrium data are plotted according to Eq. [14] to obtain the site specific equilibrium constant, $K_{3}$. The value of $K_{3}$ is substituted into Eqs. [10A] and [10B] allowing the other site specific equilibrium constants to be solved.

\section{Application of the Method to Thioredoxin Reductase}

Thioredoxin reductase has two oxidationreduction sites, an FAD and a disulfide, and catalyzes the reversible transfer of electrons between NADPH and the disulfide of thioredoxin, a small protein (21-23). In the analysis of thioredoxin reductase which follows, 
the sites of ${ }_{0 x} P_{0 x}$ in Fig. 1 correspond to ${ }_{\mathrm{FAD}} P_{(\mathrm{S}) 2}$ for thioredoxin reductase. The $E_{\mathrm{m}}$ values for the FAD and disulfide couples of thioredoxin reductase were previously determined (13). In the previous study, the binding of phenylmercuric acetate to one enzyme form was monitored which allowed solving for the four microscopic $E_{\mathrm{m}}$ values directly. The $E_{\mathrm{m}}$ values were obtained from measurements of the four enzyme forms in equilibrium with $\mathrm{NAD}^{+}$and NADH using the Nernst relationship. The criteria that $\mathrm{NAD}^{+}$and $\mathrm{NADH}$ do not bind preferentially to any of the enzyme microforms and the criteria that the enzyme microforms are in equilibrium were established in the earlier study. The Redox Scatchard analysis, described below, is of equilibrium data at $\mathrm{pH}$ 6.0. This $\mathrm{pH}$ was chosen since the difference in $E_{\mathrm{m}}$ between the FAD and disulfide couples is the greatest at this $\mathrm{pH}$ (13). The Redox Scatchard plot for titrations of thioredoxin reductase with NADH is shown in Fig. 4. The values of $K_{\alpha}$ and $K_{\beta}$, given in

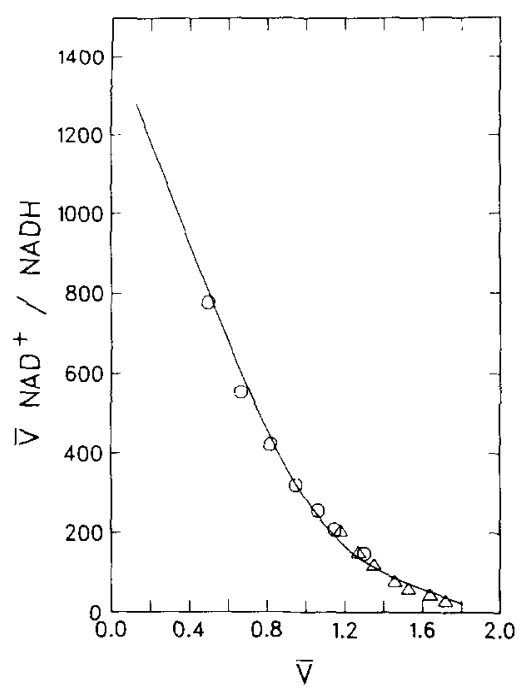

FiG. 4. Redox Scatchard plot of equilibrium data from an anaerobic titration of thioredoxin reductase with NADH. Enzyme was $17.0 \mu \mathrm{M}$ in $0.1 \mathrm{M} \mathrm{NaH}_{2} \mathrm{PO}_{4} /$ $\mathrm{K}_{2} \mathrm{HPO}_{4}-0.3 \mathrm{mM}$ EDTA, pH 6.0 at $12^{\circ} \mathrm{C}$. Two titrations were performed which differed in the initial concentration of $\mathrm{NAD}^{+}$; circles: $1.525 \mathrm{mM} \mathrm{NAD}^{+}$; triangles: $0.628 \mathrm{~mm}$ $\mathrm{NAD}^{+}$. The solid line is a computer fit to the data $\left(K_{a}\right.$ $=1455, K_{f}=55$ ).
TABLE 2

Values for the Parameters of Equations DeSCRIBING THE EQUILIBRIUM BETWEEN THE FOUR OXIDATION-REDUCTION COUPLES OF THIOREDOXIN REDUCTASE AND THE NADH/NAD ${ }^{+}$COUPLE

\begin{tabular}{lc}
\hline Constant & Value \\
\hline$K_{a}$ & 1455 \\
$K_{\beta}$ & 55 \\
Intercept & 0.00323 \\
$K_{1}\left(E_{\mathrm{m}}^{1}\right)$ & $260(-0.202 \mathrm{~V})$ \\
$K_{2}\left(E_{\mathrm{m}}^{2}\right)$ & $1195(-0.183 \mathrm{~V})$ \\
$K_{3}\left(E_{\mathrm{m}}^{3}\right)$ & $310(-0.199 \mathrm{~V})$ \\
$K_{4}\left(E_{\mathrm{m}}^{4}\right)$ & $67(-0.218 \mathrm{~V})$ \\
\hline
\end{tabular}

Note. Definitions of the constants are as follows: $K_{a}$, $K_{\beta}$ are phenomenological constants obtained from a computer fit of the data shown in Fig. 4 to Eq. [9]; intercept is the intercept of the plot shown in Fig. 6; $K_{1}, K_{2}, K_{3}$, and $K_{4}$ are the site specific equilibrium constants (see text) calculated by substituting the values $K_{\alpha}, K_{\beta}$, and intercept into Eqs. [10A] and [10B]; the $E_{\mathrm{m}}$ values are calculated from the site specific equilibrium constants using the Nernst equation. These data are from $\mathrm{NADH}$ titrations at $\mathrm{pH} 6.0$.

Table 2, were obtained from a computer fit of the data shown by the solid line in Fig. 4. The Redox Scatchard plot is an upward concave curved line indicating nonidentical $E_{\mathrm{m}}$ values of the sites and/or a negative interaction between the sites. The Redox Scatchard plots for the individual sites are shown in Figs. 5A and $B\left(\bar{v}_{1}\right.$ and $\bar{v}_{2}$ are the values of fractional reduction for the disulfide site and FAD site, respectively). The data points for neither of the site specific Redox Scatchard plots fall on a straight line indicating an interaction between the oxidation-reduction sites. The profile of the data points of the disulfide site (Fig. $5 \mathrm{~A}$ ) is different from the profile of the data points of the FAD site (Fig. 5B) showing that the sites have different $E_{\mathrm{m}}$ values. Thus, thioredoxin reductase is a protein having negative interacting/nonidentical oxidation-reduction sites. As discussed earlier, the phenomenological constants for the negative interacting/ nonidentical sites case are functions of three unequal site specific equilibrium constants 

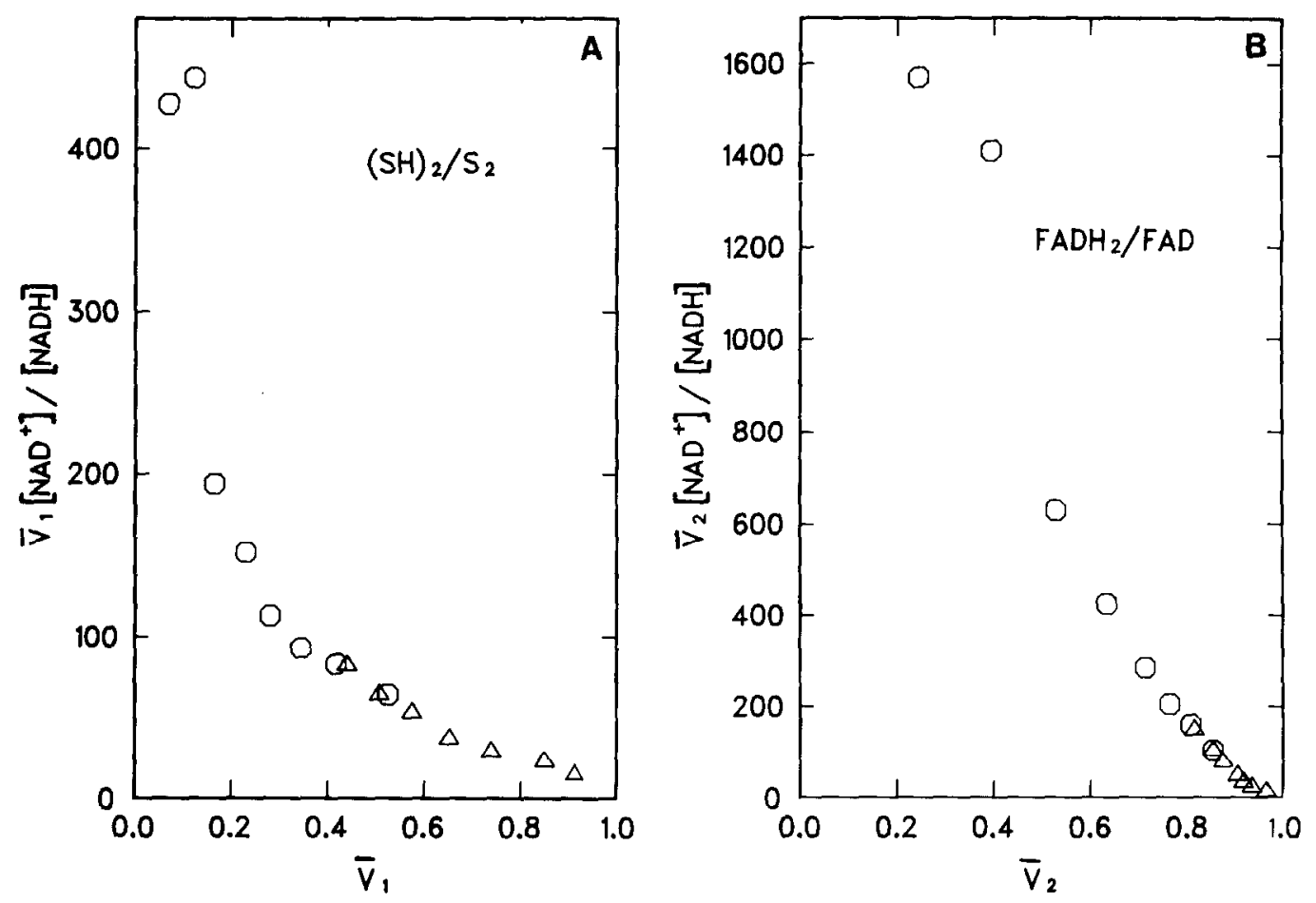

FIG. 5. Site specific Redox Scatchard plots of equilibrium data for the disulfide (A) and FAD (B) sites of thiorcdoxin reductase titrated with NADH. Conditions of the titrations and the definition of the symbols are given in the legend to Fig. 4.

(Eqs. [10A] and [10B]. The site specific equilibrium constant, $K_{3}$ (Table 2), is the reciprocal of the intercept on the ordinate (Table 2) of the plot according to Eq. [14] shown for thioredoxin reductase in Fig. 6 . The other site specific equilibrium constants are obtained by substituting the values of $K_{3}, K_{\alpha}$, and $K_{\beta}$ into Eqs. [10A] and [10B] and are given in Table 2. The site specific $E_{\mathrm{m}}$ values are obtained from the equilibrium constants using the Nernst equation and are given in Table 2 . The $E_{\mathrm{m}}$ values are within $0.004 \mathrm{~V}$ of the values determined by a different method (13). The analysis shows the FAD and disulfide couples of thioredoxin reductase to differ by approximately $0.019 \mathrm{~V}$ and have a negative interaction of about $0.016 \mathrm{~V}$ at $\mathrm{pH} 6.0$.

\section{DISCUSSION}

A graphical method to analyze interactions among oxidation-reduction sites for a two site

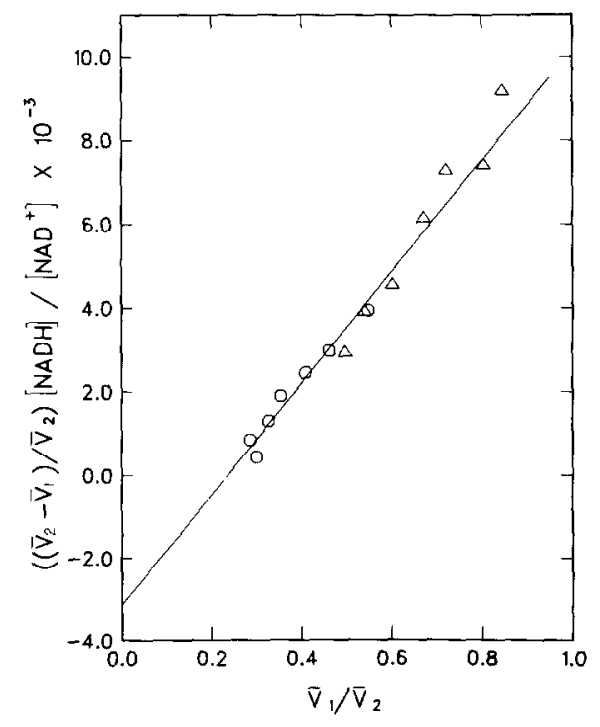

FIG. 6. Plot according to Eq. [14] for the site specific equilibrium data of thioredoxin reductase titrated with NADH. Experimental conditions and definitions of the symbols are given in the legend to Fig. 4 . The line through the data points is a linear regression analysis of the data. 
protein has been described which is analogous to the Scatchard analysis of ligand binding. In ligand binding systems, usually only total site binding can be measured and simplifying assumptions must be made to solve the site specific binding constants of even a simple two site protein. The strength of the analysis of oxidation-reduction site interactions lies in the fact that oxidation-reduction sites often have distinct physical properties conferring the ability to measure the oxidation-reduction state of individual sites in many proteins. Measurements of fractional reduction levels of individual sites in a two site protein allows a further analysis that can be combined with the Redox Scatchard analysis to solve for the specific site equilibrium constants without the need for simplifying assumptions. Hence, specific site information offers an advantage over ligand binding systems that usually allow only total site binding to be measured.

In ligand binding, extremely tight binding of a ligand requires measurements in very dilute solution to observe free ligand. In oxidation-reduction equilibria, an unfavorable equilibrium can be made more favorable by the previous addition of one species of titrant or the equilibrium can be changed by switching to a titrant having a more favorable oxidation-reduction potential, hence a further advantage of measurements of oxidation-reduction equilibria over ligand binding equilibria.

The principles of the analysis were illustrated by considering a two-site case in detail. Application of the method to thioredoxin reductase, an enzyme containing an active site disulfide and an FAD, revealed a slight negative interaction between the sites $(0.016 \mathrm{~V})$ and a difference in $E_{m}$ of $0.019 \mathrm{~V}$ for the two sites at $\mathrm{pH}$ 6.0. The values of $E_{\mathrm{m}}$ obtained from the Redox Scatchard analysis agree quite well with the values of $E_{\mathrm{m}}$ determined by a different method (13). The analysis is of equilibrium data at $\mathrm{pH} 6.0$ since the difference in $E_{\mathrm{m}}$ between the FAD and disulfide couples is the greatest at this $\mathrm{pH}$.

Oxidation-reduction equilibria among multiple sites, like multiple site ligand binding equilibria, can be described by a linear summation of hyperbolic terms with the total number of terms not exceeding the total number of sites with or without interactions among sites (1)

$$
\bar{v}=\frac{K_{i}\left[N_{\mathrm{r}}\right] /\left[N_{\mathrm{ox}}\right]}{1+K_{i}\left[N_{\mathrm{r}}\right] /\left[N_{\mathrm{ox}}\right]},
$$

where $\vec{v}$ is the average number of moles of reduced sites per mole of protein, $n$ is the total number of sites, $K_{i}$ are phenomenological constants which are functions of the site specific equilibrium constants, and $\left[N_{\mathrm{r}}\right]$ and $\left[N_{\text {ox }}\right]$ are reduced and oxidized titrant, respectively. Thus, the analysis should be applicable to proteins containing more than two sites. Site specific equilibrium measurements on a protein containing more than two sites, although more difficult to analyze than the two-site case, should give the information required to obtain the site specific equilibrium constants.

Although the function of oxidation-reduction equilibria $(\mathrm{A}+\mathrm{B} \leftarrow \mathrm{C}+\mathrm{D})$ analogous to the Scatchard function of ligand binding $(\mathrm{A}+\mathrm{B} \hookrightarrow \mathrm{C})$ has been the focus of this study, functions of the oxidation-reduction equation which are analogous to other transformations of the ligand binding equation could be useful, e.g., the Klotz plot and the Stockell plot.

\section{ACKNOWLEDGMENTS}

We are grateful for many helpful discussions with Dr. Martha Ludwig.

This research was supported by the Medical Research Service of the Veterans Administration and in part by Grant GM-21444 from the National Institute of General Medical Sciences, Public Health Service.

\section{REFERENCES}

1. Klotz, I. M., and Hunston, D. L. (1979) Arch. Biochem. Biophys. 193, 314-328.

2. George, P., and Griffith, J. S. (1959) The Enzymes (Boyer, P. D. ed.). Vol. 1, pp. 347-389, Academic Press, New York.

3. Malstrom, B. G. (1974) Q. Rev. Biophys. 6, 389-431.

4. Lanne, B., and Vanngard, T. (1978) Biochim. Biophys. Acta 501, 449-457.

5. Pecht, I., and Blatt, Y. (1979) Biochemistry 18, 2917 2922. 
6. van Gelder, B. F., and Beinert, H. (1969) Biochim. Biophys. Acta 189, 1-24.

7. Wilson, D. F., Lindsay, J. G., and Brocklehurst, E. S. (1972) Biochim. Biophys. Acta 256, 277-286.

8. Tiesjema, R. H., Muijsers, A. O., and van Gelder, B. F. (1973) Biochim. Biophys. Acta 305, 19-28.

9. Eisenstein, K. K., and Wang, J. H. (1969) J. Biol. Chem. 244, 1720-1728.

10. Farver, O., Goldberg, M., Wherland, S., and Pecht, I. (1978) Proc. Nat. Acad. Sci. USA 75, 52455249.

11. Dahlquist, F. W. (1978) in Methods in Enzymology (Hirs, C. H. W. ed.), Vol. 48, Academic Press, New York. pp. 270-299.

12. Pigiet, V. P., and Conley, R. R. (1977) J. Biol. Chem. 252, 6367-6372.

13. O'Donnell, M. E. and Williams, C. H., Jr. (1983) J. Biol. Chem., in press.

14. Clark, W. M. (1960) Oxidation-Reduction Potentials of Organic Systems, Williams \& Wilkins, Baltimore.
15. Fletcher, J. E., and Spector, A. A. (1968) Comput. Biomed. Res. 2, 164-175.

16. Scatchard, G. (1949) Ann. N. Y. Acad. Sci. 51, 660672.

17. Klotz, I. M., and Hunston, D. L. (1971) Biochemistry 10, 3065-3069.

18. Von Muralt, A. L., (1930) J. Amer. Chem. Soc. 52, 3518-3522.

19. Fletcher, J. E., Spector, A. A., and Ashbrook, J. D. (1970) Biochemistry 9, 4580-4587.

20. Larsson-Raznikiewicz, M. (1973) Arch. Biochem. Biophys. 158, 751-762.

21. Zanetti, G., and Williams, C. H., Jr. (1967) J. Biol. Chem. 242, 5232-5236.

22. Moore, E. C., Reichard, P., and Thelander, L. (1964) J. Biol. Chem. 239, 3445-3452.

23. Ronchi, S., and Williams, C. H., Jr. (1972) J. Biol. Chem. 247, 2083-2086. 